\title{
A Cross Sectional Study of Type 2 Diabetes Mellitus Comparing Different Factors between Lean Body Weight, Non Obese and Obese Patients in Western Uttar Pradesh
}

\section{Dr. Manish Shrivastav ${ }_{4}^{1}$, Dr. Nihit Kharkwal ${ }^{*} \uparrow, 2$, Dr. Alankar Tiwari ${ }^{3}$, Dr. Keshav Kumar Gupta ${ }^{4}$}

\author{
${ }^{1}$ Lala Lajpat Rai Medical College, Meerut \\ ${ }^{2}$ Lala Lajpat Rai Medical College,Meerut, Post Doctoral TraineeDepartment of Endocrinology \\ ${ }^{3}$ Post Doctoral TraineeDepartment of Endocrinology Lala Lajpat Rai Medical College, Meerut \\ 4 Professor and Head,Department of Endocrinology
}

DDOI: https://doi.org/10.15520/jcmro.v3i01.248

Accepted 13-01-2020; Received 10-11-2019; Publish Online 14-01-2020

Reviewed By:
Dr. Bhupendra Singh
Department:
Reviewer/CMRO

\section{INTRODUCTION:}

Diabetes mellitus is most common non- communicable disorder in the world. By the year 2025 over $75 \%$ of the diabetic people are expected to be in developing countries as compared to $52 \%$ in 1995 . The greatest increase will be seen in Indians of $195 \%$ from 19 million.

\footnotetext{
${ }^{\star}$ Corresponding author.

† Email: nihitkgmu@gmail.com

\begin{abstract}
Introduction- Incidence and prevalence of diabetes mellitus has been increased quite rapidly from last decade. It was thought previously that obesity is one of the major predisposing risk factor and complications of diabetes would be more common and fatal in obese persons with diabetes, but it has been shown in more recent studies that some of the factors and peculiarities are much more common in patients with lean body mass.

Material and methods- This study was done in a tertiary care centre of western Uttar Pradesh over 240 cases of type 2 diabetes mellitus that were divided into three categories in equal proportion considering their body mass index (lean, non obese and obese ). Some of the factors like urine microscopy, fasting and post prandial glucose level, glycosylated hemoglobin, lipid profile and kidney functions were studied to find out the difference in level of above mentioned factors in these groups.

Results- Fasting blood glucose, post prandial blood glucose, glycosylated hemoglobin level, and triglyceride level came to be signinifiacntly higher in lean body mass group as compared to other two groups. As far as renal status is concerned there was no significant variation in different groups of diabetes mellitus.

Conclusion- Present study showed that lean body weight type of type 2 diabetes mellitus are definitely different subset of population of type 2 diabetes mellitus with clinical characteristics different from other subtype i.e. non obese and obese in this region of country.
\end{abstract}

Key words: Hypercholesterolemia-Microproteinuria-Dyslipidemia-BodyMassIndexGlycosylated
Diabetes mellitus comprises of common metabolic disorder that share the same phenotype of hyperglycemia. Depending upon etiology of diabetes mellitus, factors contributing to hyperglycemia may include reduced insulin secretion, decreased glucose utilization by body, increased glucose production.

Two broad categories of diabetes mellitus are designated.

1. Type 1 diabetes mellitus

2. Type 2 diabetes mellitus 
Type 1 is immune mediated and there is absolute deficiency of insulin. Type 2 is generally associated with obesity in western population but in India type 2 diabetes mellitus are of (1) normal body weight, (2) obese and (3) persons with low body weight(lean) $[\mathrm{BMI}<20]$. As far as far as diabetes in India is concerned, vast majority are found to be non obese, in contrast to WHO prediction of $60-80$ percent to obese, and almost one fourth has a habitus to be called a lean $(\mathrm{BMI}<20)$ (WHO study group on diabetes mellitus, 1985). In multicentric study involving nine centers all over the country (1984-1990) the incidence of lean type 2 diabetes mellitus was observed to be 11 to 25 percent of all the diabetics' diagnosed diabetes mellitus. Epidemiological data over the past decades have shown that the pattern and profile of type 2 diabetes mellitus are very different in India compared to the west [1] In 1965 Tripathi and Kar highlighted that $27 \%$ of elderly diabetics were lean [2].following that various studies in India have reported a prevalence of low body weight/lean type 2 diabetes mellitus ranging from $1.6 \%$ to $26 \%$ [3-5] . Markers of autoimmune destruction of beta cells are absent in vast majority of these patients [5] .Homeostatic model assessments of Das et al suggest they are typical cases of type 2 diabetes and the low body weight does not reflect poor beta cell function or loss of body weight due to long st6anding uncontrolled diabetes. With this background, we will study the clinical and biochemical profile of diabetes patients in obese, non- obese and lean body habitus in western Uttar Pradesh, as there is no published data from this region. Idea of this study was to corroborate and compare the available data on low body weight type 2 diabetes mellitus in our present set up of population, whether they are real different subset of population or they do not merit any real markers for acquiring subset strategy.

\section{MATERIALS AND METHODS:}

The study was undertaken in LALA LAJPAT RAI MEDICAL COLLEGE, MEERUT in department of human metabolism and endocrinology (HME). 240 cases of type 2 diabetes mellitus ( 80 cases of lean; 80 cases of non- obese; 80 cases of obese) were selected from ambulatory patients attending HME outpatient department and admitted patients in the HME wards from November 2018 to November 2019.

\section{Inclusion criteria:}

1. All patients with type 2 diabetes mellitus irrespective of age, sex.

2. Diagnosis of diabetes mellitus was based on American Diabetes Association criteria.

3. Those given written consent.

\section{Exclusion criteria:}

1. Patients suffering from hepatic, cardoiorespiratory, endocrine and other systemic disease.

2. Pregnant female patients.
3. Known case of type 1 diabetes mellitus.

4. Patients receiving drugs causing hyperglycemia.

WHO expert committee on diabetes 2000 recommended the following concentration of glucose in venous blood estimated by normal enzymatic assay to be labeled as diabetes. Plasma glucose level is $15 \%$ higher than those of whole blood.

\begin{tabular}{|l|l|l|}
\hline Diabetes & Plasma glucose $\mathbf{m m o l} / \mathbf{l}(\mathrm{mg} / \mathrm{dl})$ & Whole blood \\
\hline Fasting & $\geq 7.0(126 \mathrm{mg} / \mathrm{dl})$ & $\geq 6.1(110 \mathrm{mg} / \mathrm{dl})$ \\
\hline 2 hours after glucose load & $\geq 11.1(200 \mathrm{mg} / \mathrm{dl})$ & $\geq 10.0(180 \mathrm{mg} / \mathrm{dl})$ \\
\hline
\end{tabular}

After diagnosing the patients as type 2 diabetes mellitus, detailed interrogation and clinical examination using questionnaire detailed at the end of dissertation. Following points were highlighted in the general examination.

1. Body mass index $(\mathrm{BMI})=$ Weight $(\mathrm{kg}) /$ Height $(\mathrm{m}) 2$

2. Blood pressure- measured in supine and standing position

Following laboratory investigations were carried out-

1. Routine urine examination with special importance of sugar, protein, pus cells and rbc

2. Fasting blood glucose and post prandial blood glucose

3. Lipid profile including- total cholesterol, LDL cholesterol, HDL cholesterol, VLDL cholesterol and triglyceride.

4. Blood urea and serum creatinine

5. Glycosylated haemoglobin

Statistical method- following method were used for statistical analysis of data of present study-

1. Descriptive study

2. Contingency coefficient for categorical variables likesex, socioeconomic status, family history and mode of presentation.

3. General linear model - multivariate with controlling for age for a set of dependent variables e.g. all the biochemical parameters across three fixed factor -lean, non obese and obese.

4. Post -hoc LSD statistic applied to examine group difference between lean, non obese and obese. 
Table 1. showing distribution of subjects

\begin{tabular}{lcllll}
\hline Type of & No.of & male & \multicolumn{3}{c}{ Female } \\
type 2 DM & cases & No. & $\%$ & No. & $\%$ \\
Lean & 80 & 52 & 65 & 28 & 35 \\
Non obese & 80 & 56 & 70 & 24 & 30 \\
Obese & 80 & 36 & 45 & 44 & 55 \\
total & 240 & 144 & 60 & 96 & 40 \\
\hline
\end{tabular}

\section{RESULTS:}

In the following section the result and their subsequent analysis of relevant clinical and other investigations features have been detailed. 80 cases of lean type 2 diabetes mellitus, 80 cases of non obese type 2 diabetes mellitus (BMI $>20.25-<25)$ and 80 cases of obese type 2 diabetes mellitus $(\mathrm{BMI}>25)$ were selected for present study. Tables 1, 2, 3, 4, $5,6,7$ and 8

Among lean cases $65 \%$ are male and $35 \%$ are female. Among non obese $70 \%$ are male and $30 \%$ are female. Among obese $45 \%$ are male and $55 \%$ cases are female.

Among lean subjects mean duration of diabetes, mean fasting and post prandial blood glucose and glycosylated hb values were 5.5 years, $226.5 \mathrm{mg} / \mathrm{dl}, 282.5 \mathrm{mg} / \mathrm{dl}$, and $9.15 \mathrm{re}-$ spectively. Among obese these values were 5.75 years, 193.1 $\mathrm{mg} / \mathrm{dl}, 248.65 \mathrm{mg} / \mathrm{dl}$ and 7.81 respectively. While among obese these were 6.1 years, $206.65 \mathrm{mg} / \mathrm{dl}, 262.7 \mathrm{mg} / \mathrm{dl}$ and 8.44 respectively.

Mean values of total cholesterol and LDL cholesterol were highest in obese subjects. While mean values of VLDL cholesterol, HDL cholesterol and triglycerides were higher in lean subjects.

Sugar in urine was present in $100 \%$ of lean patients and $90 \%$ and $95 \%$ in non obese and obese patients respectively. Microprotienuria was present in $15 \%$ of each group.

Mean values of urea and creatinine were highest in lean subjects among all the three groups.

In this analysis HDL-c, VLDL-C, urea and creatinine were not found to be significant. So next analysis was done with those dependent variables which showed significance.

\section{DISCUSSION:}

Type 2 diabetes mellitus accounts for the $85 \%$ of the people with diabetes worldwide. Development of the disease is summation of environmental insult to genetically predetermined metabolic disruption. Type 2 diabetes mellitus is characterized by the pathophysiologic abnormalities, impaired insulin secretion, peripheral insulin resistance and excessive hepatic glucose production. Obesity visceral or central is very common in type 2 diabetes mellitus. Low body weight type 2 diabetes mellitus, phenotypically a separate type of type 2 diabetes mellitus is of interest in tropical region. Characterized by its typical age presentation, altered lipid pattern along with some controversial renal involvement, more elevated fasting and post prandial blood glucose at the time of presentation of low body weight type 2 diabetes mellitus in comparison to obese and non obese type distinguishes it further. The increased mortality among lean diabetic smokers has been observed in the general population as well [6-9]. A possible explanation for the observed obesity paradox could be sarcopenic obesity, defined as the presence of high body fat with reduced or normal lean body mass [10]. Sarcopenic obesity reduces the cardiopulmonary fitness and physical functioning possibly leading to premature death and could account for the higher mortality eventually seen in individuals who are normal weight at the time of onset of diabetes mellitus [11].

C.S. Yagnik et al depicted in his study [12] the male preponderance in lean type 2 diabetes mellitus, which is comparative with the sexual distribution in present study which showed $65 \%$ male and $35 \%$ female distribution among lean type 2 diabetes mellitus.

Body mass index is the single most important predictor of low body weight type 2 diabetes mellitus and other phenotypically different types of type 2 diabetes mellitus. According to Indian authorities and WHO if the body mass index is less than 20.25, type 2 diabetes mellitus is considered as lean body weight. In the present study 17.91 is the mean BMI of type 2 diabetes mellitus belonging to low body group. This is at par with the observation of other and was significantly lower than non obese (23.01) and obese (27.44).

One important observation is that fasting blood glucose in low body type 2 diabetes mellitus is higher $(226.5 \mathrm{mg} / \mathrm{dl})$ in comparison to non obese $(193.1 \mathrm{mg} / \mathrm{dl})$ and obese ( 206.65 $\mathrm{mg} / \mathrm{dl}$ ). It is consistent with previous large series of observation by S. Das et al [13], B.K. Sahay and K.Kannan, V. Seshaish et al [14] and Sameer Banerjee. Post prandial blood glucose was also higher in case of lean type 2 diabetes mellitus as compared to non obese and obese $(282.85 \mathrm{mg} / \mathrm{dl} \mathrm{vs}$ $248.65 \mathrm{mg} / \mathrm{dl}$ vs $262.7 \mathrm{mg} / \mathrm{dl}$ ). These observation are associated with significantly high mean glycosylated hemoglobin level in lean type 2 diabetes mellitus than non obese and obese cases (9.15 vs 7.81 vs 8.44 ). This data is consistent bwith studies of S Das and V Sidharta et al [15].In this study using general linear model multivariate with controlling for age and POST HOC LSD, FBG, PPBG and glycosylated hemoglobin was found to be significantly increased in lean compared to non obese and obese. All the above observation suggest that low body weight type 2 patients are having less insulin secretion either4 in fasting state or post prandial state and it is also evident that day to day regulation of insulin secretion is also defective.

Hypercholesterolemia i.e. cholesterol level $>240 \mathrm{mg} / \mathrm{dl}$ $(10 \%)$ in contrast to hypertriglyceridemia i.e. triglyceride level $>200 \mathrm{mg} / \mathrm{dl}(35 \%)$ was less common in low body weight type 2 diabetes mellitus but HDL cholesterol value 
Table 2. Base line data showing mean duration of diabetes in years, mean fasting and post prandial blood glucose and mean glycosylated hb\% value- .

\begin{tabular}{llll}
\hline Data & Lean & Non obese & obese \\
Mean duration of diabetes in years & $5.5 \pm 3.45$ & $5.75 \pm 3.13$ & $6.1 \pm 2.19$ \\
Fasting blood glucose mg/dl & $226.5 \pm 17.78$ & $193.1 \pm 29.0$ & $206.65 \pm 29.18$ \\
Post prandial blood glucose mg/dl & $282.5 \pm 20.74$ & $248.65 \pm 36.13$ & $262.7 \pm 33.01$ \\
Glycosylated hemoglobin HbA1C & $9.15 \pm 1.01$ & $7.81 \pm 0.64$ & $8.44 \pm 0.952$ \\
\hline
\end{tabular}

Table 3. Mean values of lipid profile in lean, non obese and obese type 2 diabetes mellitus-

\begin{tabular}{llll}
\hline Lipid profile $(\mathrm{mg} / \mathrm{dl})$ & lean & Non obese & Obese \\
Total cholesterol & $207.9 \pm 18.4$ & $227.5 \pm 22.0$ & $229.2 \pm 19.9$ \\
LDL cholesterol & $122.85 \pm 16.8$ & $149.4 \pm 25.0$ & $150.7 \pm 21.5$ \\
VLDL cholesterol & $38.1 \pm 4.7$ & $35.3 \pm 5.9$ & $33.8 \pm 3.9$ \\
HDL cholesterol & $46.0 \pm 6.8$ & $42.1 \pm 5.6$ & $44.1 \pm 8.6$ \\
triglycerides & $186.1 \pm 20.5$ & $170.9 \pm 24.7$ & $168.5 \pm 18.2$ \\
\hline
\end{tabular}

Table 4. urinary changes in type 2 diabetes mellitus

\begin{tabular}{lllllll}
\hline Data & lean & \multicolumn{5}{l}{$\begin{array}{l}\text { Non } \\
\text { obese }\end{array}$} \\
& No. & $\%$ & No. & $\%$ & No. & $\%$ \\
Sugar & 80 & 100 & 72 & 90 & 76 & 95 \\
Microproteinuria & 12 & 15 & 12 & 15 & 12 & 15 \\
Overt proteinuria & 4 & 5 & 8 & 10 & 16 & 20 \\
Pus cells & 4 & 5 & - & & 4 & 5 \\
rbc & - & & 2 & 5 & - & \\
\hline
\end{tabular}

Table 5. mean values of blood urea and creatinine

\begin{tabular}{lll}
\hline Type 2 diabetes mellitus & Blood urea & Serum creatinine \\
Lean & $30.2 \pm 17.0$ & $1.18 \pm 0.55$ \\
Non obese & $25.4 \pm 8.4$ & $1.12 \pm 0.30$ \\
obese & $25.2 \pm 14.3$ & $1.13 \pm 0.66$ \\
\hline
\end{tabular}

Table 6. General linear model multivariate controlling for age for a set of dependent variables across three fixed factors (lean, non obese and obese)

\begin{tabular}{|c|c|c|c|c|c|c|}
\hline & Mean value of lean & Mean value of non obese & $\begin{array}{l}\text { Mean value of } \\
\text { obese }\end{array}$ & $\begin{array}{l}\mathrm{F} \\
\text { value }\end{array}$ & $\begin{array}{l}\text { signifi- } \\
\text { cance }\end{array}$ & remark \\
\hline FBG & $226.5 \pm 17.7$ & $193.1 \pm 29.0$ & $206.6 \pm 29.1$ & 8.53 & 0.001 & Significant \\
\hline PPBG & $282.8 \pm 20.7$ & $248.6 \pm 36.1$ & $262.7 \pm 33.0$ & 6.77 & 0.002 & Significant \\
\hline $\mathrm{TC}$ & $207.9 \pm 18.4$ & $227.5 \pm 22.03$ & $229.2 \pm 19.9$ & 5.66 & 0.006 & Significant \\
\hline LDL-C & $122.8 \pm 16.8$ & 149.425 .01 & $150.7 \pm 21.5$ & 9.16 & 0.001 & Significant \\
\hline HDL-C & $46.0 \pm 6.87$ & $42.1 \pm 5.68$ & $44.1 \pm 8.6$ & 1.39 & 0.25 & $\begin{array}{l}\text { Non - } \\
\text { significant }\end{array}$ \\
\hline VLDL & $38.1 \pm 4.70$ & $35.3 \pm 5.99$ & $33.8 \pm 3.9$ & 3.43 & 0.06 & Non significant \\
\hline TG & $186.1 \pm 20.5$ & $170.9 \pm 24.7$ & $168.5 \pm 18.2$ & 4.01 & 0.02 & Significant \\
\hline UREA & $30.2 \pm 17.04$ & $25.4 \pm 8.47$ & $25.2 \pm 14.3$ & 0.83 & 0.44 & Non-significant \\
\hline $\begin{array}{l}\text { CREATI- } \\
\text { NINE }\end{array}$ & $1.18 \pm 0.55$ & $1.12 \pm 0.30$ & $1.13 \pm 0.66$ & 0.11 & 0.89 & Nosignificant \\
\hline
\end{tabular}

Table 7. post HOC LSD statistics applied to examine group difference between lean and non-obese

\begin{tabular}{lllllll}
\hline Dependent variables & weight type (i) & Weight type $(\mathrm{j})$ & Mean difference(i-j) & Std. error & significance & Remark \\
FBG & lean & Non-obese & 33.4 & 8.18 & 0.001 & significant \\
PPBG & lean & Non-obese & 34.2 & 9.70 & 0.001 & significant \\
TC & lean & Non-obese & -19.6 & 6.38 & 0.003 & significant \\
LDL-C & lean & Non-obese & -26.5 & 6.77 & 0.001 & significant \\
TG & lean & Non-obese & 15.2 & 6.75 & 0.028 & significant \\
\hline
\end{tabular}


Table 8. post HOC LSD statistics applied to examine group difference between lean and obese

\begin{tabular}{|c|c|c|c|c|c|c|}
\hline Dependent variables & weight type (i) & weight type $(\mathrm{j})$ & Mean difference(i-j) & Std. error & signifiacnce & Remark \\
\hline FBG & lean & Obese & 19.8 & 8.18 & 0.018 & significant \\
\hline PPBG & lean & Obese & 20.1 & 9.70 & 0.042 & significant \\
\hline $\mathrm{TC}$ & lean & Obese & -21.3 & 6.38 & 0.001 & significant \\
\hline LDL-C & lean & Obese & -27.8 & 6.77 & 0.001 & significant \\
\hline TG & lean & Obese & 17.6 & 6.75 . & 0.011 & significant \\
\hline
\end{tabular}

was not significantly variable in the low body weight as compared to non obese and obese. Lowe incidence of hypercholesterolemia and relatively higher incidence of hypertriglyceridemia in low body weight was also found in studies of K. Kannan, C.S. Yagnik et al [12].

The increased incidence of raised blood urea and serum creatinine was found more in low body weight as compared to other two groups. It is consistent with finding by Sidhartha Das. But this difference was not significant.

The most characteristics criteria in low body weight type 2 diabetes mellitus i.e. lower body mass index has got increased fasting and post prandial blood sugar values in comparison with non obese and obese patients which reflect decreased beta cell reserve having lesser amount of endogenous insulin secretion. Hypertriglyceridemia, reflecting lower body insulin status is characteristics of low body weight type 2 diabetes and all these findings came out to be significant in our study also.

\section{REFERENCES}

1. Hoet JJ, Tripathy BB. Report of the international workshop on types of diabetes peculiar to the tropics. Diabetes care. 1996;19:1014.

2. Tripathy BB, Kar BC. Observations and clinical patterns of diabetes in India. Diabetes 1965;14:404-12. 3. Samal KC, Das S, Agarwal BN, Panda NC, Tripathy BB. Nutritional status and profile of NIDDM of recent onset. J Diab Assoc India,. 1998;28:99-101.

4. Ramchandran A , Snehalatha C, Latha E , Vijay V, Vishwanathan M. Rising prevalence of NIDDM in urban population in India. Diabetologia. 1997;40:232-7. 5. Mohan V, Vijayaprabha R, Rema M, premalatha G, Poongothai S, Deepa R et al. clinical profile of lean NIDDM in south India. Diabetes Res Clin Pract. 1997;38:101-8. 6. Zheng W, McLerran DF Rolland B, Zhang X, Inouse M, Matsuo K, He J, Gupta PC, Ramdas K, Tsugane S et al. Association between body mass index and risk of death in more than 1 million Asians. N Engl J Med. 2011;363:719-29. 7. Manson JE, Willett WC, Stamfer MJ, Colditz GA, Hunter DJ, Hankinson SE, Hennekens CH, Speizer FE. Body weight and mortality among women. N Engl J Med. 1995;333:677-85. 8. Pishon T, Boeing H, Hoffemann K, B ergmann M, Schulze MB, Overvad K, Van der Schouw YT, Spencer E, Moons KG, Tjonneland A. General and abdominal adiposity and risk of deathy in Europe. N Engl J Med.2008;359:2105-120. 9. Manson JE, Stamfer MJ, Hennekens CH, Willet WC. Body weight and longevity. A Reassesment. JAMA. 1987;257:353-58. 10. FloerzH, Castillo-Floerz S. Beyond the obesity paradox in diabetes: fitness, fatness and mortality. JAMA.2012;308:619-20. 11. Zamboni M, Maxzzali G, Zoico E, Harris TB, Meigs JB,Di Francesco V, Fantin F, Bissoli L, Bosello O. HJealth consequences of obesity in the elderly: a review of four unresolved questions. Int J Obes (Lond). 2005;29:1011-29.
12. Yagnik CS et al . Aossiacition of obesity with clinical, biochemical, metabolic and endocrine measurement in newly diagnosed NIDDM patients- NovoNordisk Diabetes update. Kapur A Ed. 1993, Proceedings Health care communication 139-46.

13. Das S, Samuel SC, Baliarsinha AK, Tripathy BB. Lean (underweight) NIDDM- Peculiarities and differences in metabolic and hormonal status- A pilot study. J A ssc Physicians India. 1995;43:339-42.

14. Seshaiah Vet al: Lipid profile and other risk factors associated with vascular complications in NIDDM- Lipid India ILIB Oct-Dec $1996: 10-15$.

15. Das Siddharta: Lean NIDDM: An independent entity-in Novonordisk Diabetes updates proceedings Ed. Kapur A. Health Care Communication; Bombay 1993:153-9. 